\title{
Multi-task Domain Adaptation for Sequence Tagging
}

\author{
Nanyun Peng and Mark Dredze \\ Human Language Technology Center of Excellence \\ Center for Language and Speech Processing \\ Johns Hopkins University, Baltimore, MD, 21218 \\ npenglejhu.edu, mdredzedcs.jhu.edu
}

\begin{abstract}
Many domain adaptation approaches rely on learning cross domain shared representations to transfer the knowledge learned in one domain to other domains. Traditional domain adaptation only considers adapting for one task. In this paper, we explore multi-task representation learning under the domain adaptation scenario. We propose a neural network framework that supports domain adaptation for multiple tasks simultaneously, and learns shared representations that better generalize for domain adaptation. We apply the proposed framework to domain adaptation for sequence tagging problems considering two tasks: Chinese word segmentation and named entity recognition. Experiments show that multi-task domain adaptation works better than disjoint domain adaptation for each task, and achieves the state-of-the-art results for both tasks in the social media domain.
\end{abstract}

\section{Introduction}

Many natural language processing tasks have abundant annotations in formal domain (news articles) but suffer a significant performance drop when applied to a new domain, where only a small number of annotated examples are available. The idea behind domain adaptation is to leverage annotations from high-resource (source) domains to improve predictions in low-resource (target) domains by training a predictor for a single task across different domains.

Domain adaptation work tends to focus on changes in data distributions, e.g. different words are used in each domain. Domain adaptation methods include unsupervised (Blitzer et al.,
2006) and supervised (Daumé III, 2007) variants, depending on whether there exists no or some training data in the target domain. This paper considers the case of supervised domain adaptation, where we have a limited amount of target domain training data, but much more training data in a source domain.

Work on domain adaptation mostly follows two approaches: parameter tying (i.e. linking similar features during learning) (Dredze and Crammer, 2008; Daumé III, 2007, 2009; Finkel and Manning, 2009; Kumar et al., 2010; Dredze et al., 2010), and learning cross domain representations (Blitzer et al., 2006, 2007; Glorot et al., 2011; Chen et al., 2012; Yang and Eisenstein, 2015). Often times, domain adaptation is formulated as learning a single model for the same task across domains, although with a focus on maximizing target domain performance. This is similar in spirit to multi-task learning (MTL) (Caruana, 1997) which jointly learns models for several tasks, for example. learning a single data representation common to each task (Ando and Zhang, 2005; Collobert et al., 2011; Liu et al., 2016c; Peng and Dredze, 2016; Yang et al., 2016; Liu et al., 2016a). Given the similarity between domain adaptation and MTL, it is natural to ask: can domain adaptation benefit from jointly learning across several tasks?

This paper investigates how MTL can induce better representations for domain adaptation. There are several benefits. First, learning multiple tasks provides more training data for learning. Second, MTL provides a better inductive learning bias so that the learned representations better generalize. Third, considering several tasks in domain adaptation opens up the opportunities to adapt from a different domain and a different task, a mismatch setting which has not previously been explored. We present a representation learning 
framework based on MTL that incorporates parameter tying strategies common in domain adaptation. Our framework is based on a bidirectional long short-term memory network with a conditional random fields (BiLSTM-CRFs) (Lample et al., 2016) for sequence tagging. We consider sequence tagging problem since they are common in NLP applications and have been demonstrated to benefit from learning representations (Lample et al., 2016; Yang et al., 2016; Peng and Dredze, 2016; Ma and Hovy, 2016).

This paper makes the following contributions:

- A neural MTL domain adaptation framework that considers several tasks simultaneously when doing domain adaptation.

- A new domain/task mismatch setting: where you have two datasets from two different, but related domains and tasks.

- State-of-the-art results on Chinese word segmentation and named entity recognition in social media data.

\section{Model}

We begin with a brief overview of our model, and then instantiate each layer with specific neural architectures to conduct multi-task domain adaptation for sequence tagging. Figure 1 summarizes the entire model presented in this section.

A representation learner that is shared across all domains and tasks, and learns robust data representations for features. This feeds a domain projection layer, with one projection for each domain that transforms the learned representations for different domains into the same shared space. As a result, the final layer of task specific models, which learns feature weights for different tasks, can be shared across domains since the learned representations (features) for different domains are now in the same space. The framework is flexible in both the number of tasks and domains. Increasing the number of domains linearly increases domain projection parameters, with the number of other model parameters unchanged. Similarly, increasing the number of tasks only linearly increases the number of task specific model parameters. If there is only one domain, then the framework reduces to a multi-task learning framework, and similarly, the framework reduces to a standard domain adaptation framework if there is only one task.

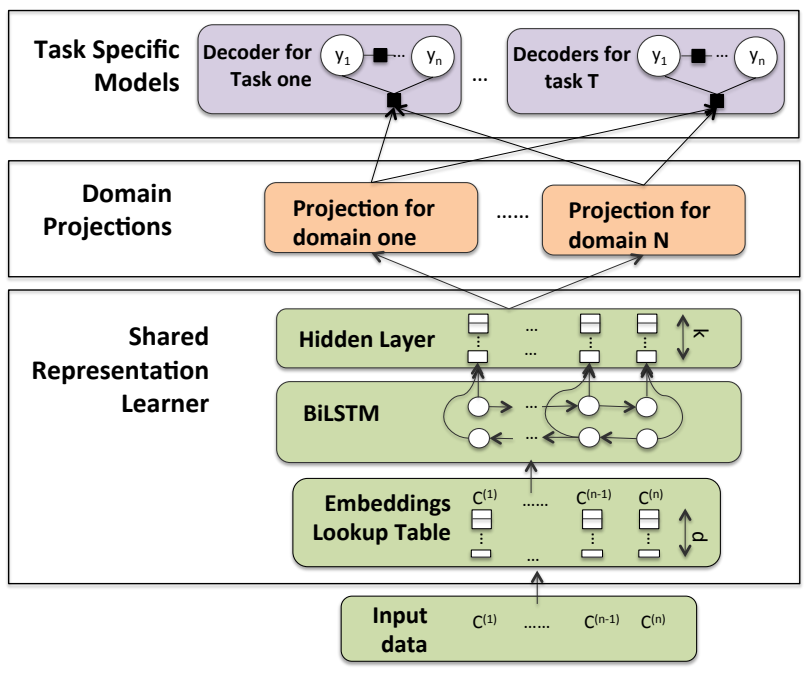

Figure 1: An overview of our proposed model framework. The bottom layer is shared by all tasks and domains. The domain projections contain one projection per domain and the task specific models (top layer) contain one model per task.

The shared representation learner, domain projections and task specific models can be instantiated based on the application. In this paper, we focus on sequence tagging problems. We now introduce our instantiated neural architecture for multitask domain adaptation for sequence tagging.

\subsection{BiLSTM for representation learning}

Long short-term memory (LSTM) (Hochreiter and Schmidhuber, 1997) is a type of recurrent neural network (RNN) that models interdependencies in sequential data. It addresses the vanishing or exploding gradients (Bengio et al., 1994; Pascanu et al., 2013) problems of vanilla RNNs by using a series of gates (input, forget and output gates) to control how memory is propagated in the hidden states of the model, and thus effectively captures long-distance dependencies between the inputs.

Many NLP applications use bi-directional LSTMs (BiLSTM) (Dyer et al., 2015) to scan both left-to-right and right-to-left, which capture left and right context. The hidden vectors produced by both LSTMs are concatenated to form the final output vector $h_{t}=\overrightarrow{h_{t}} \oplus \overleftarrow{h_{t}}$. BiLSTMs have become a common building block for learning representations in NLP and have achieved impressive performance in problems such as sequence tagging (Lample et al., 2016; Yang et al., 2016; Ma and Hovy, 2016), relation classification (Xu et al., 2015; Zhang et al., 2015), and syntactic parsing (Kiperwasser and Goldberg, 2016; Cross 
and Huang, 2016). We use a BiLSTM as our representation learner. It produces a hidden vector for each token in the sentence, which we denote as:

$$
h_{t}=\operatorname{BiLSTM}\left(x_{1: n}, t\right)
$$

where $x_{1: n}$ denotes the whole input sequence of length $n$, and $t$ denotes the $t$-th position. The representation for the whole sequence is thus denoted as $\boldsymbol{h}=h_{1: n}$.

\subsection{Domain Projections}

Domain adaptation requires learning a shared representation that generalizes across domains. Ideally, parameter estimation of the BiLSTM should learn to produce such robust features. However, this may place a heavy burden on the BiLSTM; it does not know the identity of each domain yet must still learns how to map two heterogeneous input types to the same representation. To reduce this burden, we introduce a domain projection layer, which relies on explicit domain specific transformation functions to produce shared representations. We place this transformation between the representation learner and the task specific predictor to alleviates pressure on the representation learner to learn cross domain representations. Note that the domain projection layer works jointly with the representation learner to produce shared representations. We experiment with two simple strategies for domain projections which are based on previous lines of work in domain adaptation.

\subsubsection{Domain Masks}

The first strategy is inspired by Daumé III (2007) and Yang and Hospedales (2015), which split the representations into several regions, with one region shared among domains, and others specific for each domain. As a result, the BiLSTM representation learner will learn to put the features that are suitable to be shared across domains into the shared region, and domain specific features to the corresponding region for the domain.

We implement this strategy by defining domain masks $\boldsymbol{m}_{d}$, which is a vector for the $d$ th domain. The mask $\boldsymbol{m}_{d}$ has value 1 for the effective dimensions of domain $d$ and domain shared region, and 0 for all other dimensions. For example, assume we have two domains and a $k$ dimensional hidden vector for features, the first $k / 3$-dimensions is shared between the two domains, while the $k / 3+1$ to $2 k / 3$ dimensions are used only for domain 1 , and the remaining dimensions for domain 2. The mask for domain 1 and domain 2 would be:

$$
\boldsymbol{m}_{1}=[\overrightarrow{1}, \overrightarrow{1}, \overrightarrow{0}], \quad \boldsymbol{m}_{2}=[\overrightarrow{1}, \overrightarrow{0}, \overrightarrow{1}] .
$$

We can then apply these masks directly to the hidden vectors $\boldsymbol{h}$ learned by the BiLSTM to produce a projected hidden state $\hat{\boldsymbol{h}}$ :

$$
\hat{\boldsymbol{h}}=\boldsymbol{m}_{d} \odot \boldsymbol{h},
$$

where $\odot$ denotes element-wise multiplication. Since only a subset of the dimensions are used as features in each domain, the BiLSTM will be encouraged to learn to partition the dimensions of the output hidden vectors into domains.

Note that in Daumé III (2007), the domain masks operate on hand engineered features, thus only affect feature weights. However, here the domain masks will change the parameters learned in BiLSTMs as well, changing the learned features. Therefore, training data from one domain will also change the other domains' representation. When we jointly train with data from all domains, the model has to balance the training objectives for all domains simultaneously.

\subsubsection{Linear Projection}

The second domain adaptation strategy we explore is a linear transformation to each domain, denoted as $T_{d}$. Given a $k$-dimensional vector representation $\boldsymbol{h}, T_{d}$ is a $k \times k$ matrix that projects the learned BiLSTM hidden vector to a common space that can be used by a shared task specific model. We use the transformation:

$$
\hat{\boldsymbol{h}}=T_{d} \boldsymbol{h} .
$$

We learn $T_{d}$ for each domain jointly with other model parameters. While this model has greater freedom in learning representations across domains, it relies on the training data to learn a good transformation, and does not explicitly partition the representations into domain regions.

\subsection{Task Specific Neural-CRF Models}

Multi-task domain adaptation simultaneously considers several tasks adapting domains since the related tasks would help induce more robust data representations for domain adaptation. Additionally, it enables leveraging more data to learn better domain projections. The goal of a task specific model is to learn parameters to project the shared 
representations to the desired outputs for the corresponding task. Different tasks that define different output spaces need separate task specific models.

For our applications to sequence tagging problems, we choose Conditional Random Fields (CRFs) (Lafferty et al., 2001) as task specific models, since it is widely used in previous work and is shown to benefit from learning representations (Peng and Dredze, 2015; Lample et al., 2016; Ma and Hovy, 2016). These "Neural-CRFs" define the conditional probability of a sequence of labels given the input as:

$p\left(\boldsymbol{y}^{k} \mid \boldsymbol{x}^{k} ; W\right)=\frac{\prod_{i=1}^{n} \exp \left(W^{T} F\left(y_{i-1}^{k}, y_{i}^{k}, \psi\left(\boldsymbol{x}^{k}\right)\right)\right)}{Z^{k}}$ where $i$ indexes the position in the sequence, $F$ is the feature function, and $\psi\left(\boldsymbol{x}^{k}\right)$ defines a transformation of the original input, in our case $\psi\left(\boldsymbol{x}^{k}\right)=$ $\operatorname{BiLSTM}\left(\boldsymbol{x}^{k}\right) . \quad Z^{k}$ is the partition function defined as:

$$
Z^{k}=\sum_{\boldsymbol{y} \in \mathcal{Y}} \prod_{i=1}^{n} \exp \left(W^{T} F\left(y_{i-1}^{k}, y_{i}^{k}, \psi\left(\boldsymbol{x}^{k}\right)\right)\right) .
$$

\subsubsection{Sharing Task Specific Models}

We could create a CRF decoder for each task and domain. This is the practice of some (Yang and Hospedales, 2015) who consider domain adaptation, or multi-domain learning, a special case of MTL, and learn separate models for the same task from different domains.

Instead, we argue that learning a single model for a task regardless of the number of domains draws strong connections to the traditional domain adaptation literature. It enjoys the benefit of increasing the amount of training data for each task by considering different domains, and better handles the problem of shifts in data distributions by explicitly considering different domains. Therefore, we use a single CRF per task, shared across all domains.

\section{Parameter Estimation}

The proposed neural architecture for multi-task domain adaptation can be trained end-to-end by maximizing data log-likelihood. As there are $D \times T^{1}$ datasets, the final loss function is a linear combination of the log-likelihood of each dataset. For simplicity, we give each dataset equal weight when forming the linear combination.

\footnotetext{
${ }^{1} D$ denotes the number of domains and $T$ the number of tasks
}

Training Model training is a straightforward application of gradient based back-propagation. We use alternating optimization among each dataset with stochastic gradient descent (SGD). To prevent training from skewing the model to a specific dataset due to the optimization order, we subsample the number of instances used in each epoch with a fraction $\lambda$ w.r.t. the smallest dataset size, which is tuned as a hyper-parameter on development data. A separate learning rate is tuned for each dataset, and we decay the learning rate when results on development data do not improve after 5 consecutive epochs. We train for up to 30 ,epochs and use early stopping (Caruana et al., 2001; Graves et al., 2013) as measured on development data. We select the best model for each dataset based on hyper-parameter tuning. We use dropout on the embeddings and the BiLSTM output vectors as in Ma and Hovy (2016).

Initialization We use pre-trained Chinese embeddings provided by Peng and Dredze (2015) with dimension 100. All other model parameters are initialized uniformly at random in the range of $[-1,1]$.

Inference For training the CRFs, we use marginal inference and maximize the marginal probabilities of the labels in the training data. At test time, the label sequence with highest conditional probability $y^{*}=\arg \max p(y \mid x ; \Omega)$ is obtained by MAP inference.

Hyper-parameters Our hyper-parameters include the initial learning rate (per dataset, in the range of $[0.005,0.01,0.02])$, the dropout rate for the input embedding and the hidden vectors (in the range of $[0,0.1,0.2]$ ), and the subsample coefficient for each setting (in the range of $[5,10,15]$ ). We tune these hyper-parameter using beam search on development data. For convenience, the embedding and the LSTM hidden vector dimensions are set to 100 and 150 respectively.

\section{Experimental Setup}

We test the effectiveness of the multi-task domain adaptation framework on two sequence tagging problems: Chinese word segmentation (CWS) and named entity recognition (NER). We consider two domains: news and social media, with news the source domain and social media the target domain. 


\begin{tabular}{cccc}
\hline Dataset & \#Train & \#Dev & \#Test \\
\hline SighanCWS & 39,567 & 4,396 & 4,278 \\
\hline SighanNER & 16,814 & 1,868 & 4,636 \\
\hline WeiboCWS & 1,600 & 200 & 200 \\
\hline WeiboNER & 1,350 & 270 & 270 \\
\hline
\end{tabular}

Table 1: Datasets statistics.

\subsection{Datasets}

We consider two domains: news and social media for the two tasks: CWS and NER. This results in four datasets: news CWS data comes from the SIGHAN 2005 shared task (SighanCWS) (Emerson, 2005), news NER data comes from the SIGHAN 2006 shared task (SighanNER) (Levow, 2006), social CWS data (WeiboSeg) created by Zhang et al. (2013), and social NER data (WeiboNER) created by Peng and Dredze (2015).

Both SighanCWS and SighanNER contain several portions ${ }^{2}$; we use those for simplified Chinese (PKU and MSR respectively). The datasets do not have development data, so we hold out the last $10 \%$ of training data for development. Sighan$N E R$ contains three entity types (person, organization and location), while WeiboNER is annotated with four entity types (person, organization, location and geo-political entity), including named and nominal mentions. To match the two tag sets, we only use named mentions in WeiboNER and merge geo-political entities and locations. The 2000 annotated instances in WeiboSeg were meant only for evaluation, so we split the data ourselves using an 8:1:1 split for training, development, and test. Hyper-parameters are tuned on the development data and we report the precision, recall, and F1 score on the test portion. Detailed data statistics is shown in Table 1.

\subsection{Baselines}

We consider two baselines common in domain adaptation experiments. The first baseline only considers a single dataset at a time (separate) by training separate models just on in-domain training data. The second baseline (mix) uses out-ofdomain training data for the same task by mixing it with the in-domain data. For both the baselines, we use the BiLSTM-CRFs neural architec-

\footnotetext{
${ }^{2}$ The portions are annotated by different institutes, and cover both traditional and simplified Chinese
}

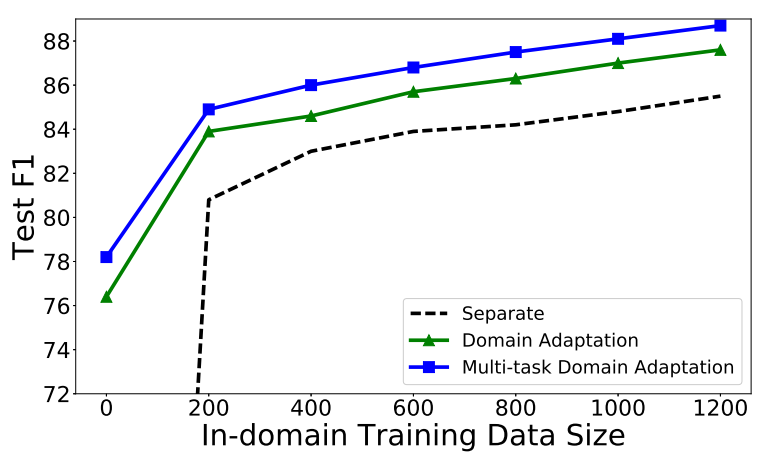

(a)

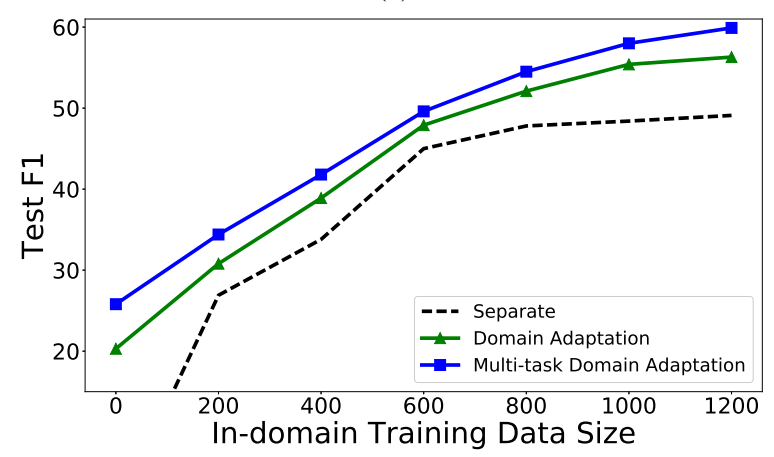

(b)

Figure 2: The effect of training data size on social media CWS (top) and NER (bottom) tasks. With more in-domain training data we see diminishing returns from domain adaptation. Our proposed multi-task domain adaptation framework is also applicable for unsupervised domain adaptation (with no in-domain training data).

ture (Lample et al., 2016), which achieved stateof-the-art results on NER and other sequence tagging tasks (Peng and Dredze, 2016; Ma and Hovy, 2016; Yang et al., 2016).

\section{Experimental Results}

\subsection{Main Results}

Table 2 presents the results for domain adaptation to the target domain (social media) test data. The baseline method Mix improves over Separate as it benefits from the increased training data. The single task domain adaptation models are a special case of the proposed multi-task domain adaptation framework: with only one task specific model in the top layer (CWS or NER). Both of our approaches (domain mask and linear projection) improve over the baseline methods. Knowing the domain of the training data helps the model better learn effective representations. Finally, we see further improvements in the multi-task domain adaptation setting. By considering additional tasks in 


\begin{tabular}{l|l|ccc|ccc}
\hline \multirow{2}{*}{ Settings } & \multicolumn{1}{|c|}{ Datasets } & \multicolumn{3}{c|}{ CWS } & \multicolumn{3}{c}{ NER } \\
& Methods & Prec & Recall & F1 & Prec & Recall & F1 \\
\hline \multirow{2}{*}{ Baseline } & Separate & 86.2 & 85.7 & 86.0 & 57.2 & 42.1 & 48.5 \\
& Mix & 87.0 & 86.1 & 86.5 & 60.9 & 44.0 & 51.1 \\
\hline \multirow{2}{*}{ Domain Adapt } & Domain Mask & 88.7 & 87.1 & 87.9 & 68.2 & 48.6 & 56.8 \\
& Linear Projection & 88.0 & 87.5 & 87.7 & 73.3 & 45.8 & 56.4 \\
\hline \multirow{2}{*}{ Multi-task DA } & Domain Mask & $\mathbf{8 9 . 7}$ & 88.3 & $\mathbf{8 9 . 0}$ & 60.2 & $\mathbf{5 2 . 3}$ & $\mathbf{5 9 . 9}$ \\
& Linear Projection & 89.1 & $\mathbf{8 8 . 6}$ & 88.9 & $\mathbf{6 8 . 6}$ & 49.5 & 57.5 \\
\hline
\end{tabular}

Table 2: Test results for CWS and Chinese NER on the target social media domain. The first two rows are baselines (Section 4.2,) followed by two domain adaptation models that only considers one task a time. The last two rows are the proposed multi-task domain adaptation framework building upon the two domain adaptation models, respectively. Domain adaptation models leverage out-of-domain training data and significantly improve over the Separate baseline, as well as the Mix baseline which trains with the out-of-domain data without considering domain shift. Multi-task domain adaptation further significantly improves over traditional domain adaptation on both domain adaptation models and achieved the new state-of-the-art results on the two tasks.

addition to domains, we achieve new state-of-theart results on the two tasks. We compare to the best published results from Zhang et al. (2013) and Peng and Dredze (2016) with F1 scores of $87.5 \%$ (CWS) and 55.3\% (NER), respectively.

Statistical Significance We measures statistical significance using McNemars chi-square test (McNemar, 1947) for paired significant test. We treated the predicted spans (not tokens) that agreed with the ground truth as positive, otherwise negative. For the NER task, we only count the spans that corresponds to named entities. We compare the best baseline (mix) and the two domain adaptation models, as well as between the domain adaptation models and their multi-task domain adaptation counterpart. Both the domain adaptation models significantly improved over the mix baseline $(p<0.01)$, and the multi-task domain adaptation methods significantly improved over their single task domain adaptation counterpart ( $p<$ 0.01 ). We cannot conduct paired significance tests with the best published results since we do not have access to their outputs.

\subsection{In-domain Training Data}

We also conducted several experiments to show the flexibility of our multi-task domain adaptation framework and analyze the behavior of the models by varying the training data.

We first consider the effect of in-domain training data size. Figure 2 shows the test F1 for the Separate baseline which only considers in-domain training data compared with both a single-task do- main adaptation model and a multi-task domain adaptation model. For simplicity, we only show the curve for the Domain Mask variant. As expected, we observe diminishing returns with additional in-domain training data on both tasks, but domain adaptation and multi-task domain adaptation methods suffer less from the diminishing return, especially on the NER task (Figure 2a). The curves for domain adaptation and multi-task domain adaptation also appear to be smoother, as they leverage more data to learn input representations, and thus are more robust.

When we have no in-domain training data, the problem reduces to unsupervised domain adaptation. Our framework applies here as well, and multi-task domain adaptation achieves performance close to the Separate baseline with only 200 in-domain training examples.

\subsection{Model Variations}

The multi-task domain adaptation framework is flexible regarding the number of domains and tasks, thus the number of datasets. Table 3 shows the results for several model variations, grouped by the number of training datasets. With one dataset, it is just the standard supervised learning setting, which reduces to our Separate baseline.

With two datasets, the framework can do multitask learning (with two datasets from the same domain but different tasks), single task domain adaptation (with two datasets for the same task but from different domains), and a novel mismatch setting (with two datasets from both different domains and different tasks). As shown in the second 


\begin{tabular}{l|l|ccc|ccc}
\hline \multirow{2}{*}{ Dataset Numbers } & \multicolumn{1}{|c|}{ Datasets } & \multicolumn{3}{c|}{ CWS } & \multicolumn{3}{c}{ NER } \\
& Methods & Prec & Recall & F1 & Prec & Recall & F1 \\
\hline One Dataset & Separate & 86.2 & 85.7 & 86.0 & 57.2 & 42.1 & 48.5 \\
\hline \multirow{3}{*}{ Two Datasets } & Multi-task & 87.7 & 86.2 & 86.9 & 59.1 & 44.9 & 51.1 \\
& Domain Adaptation & 88.7 & 87.1 & 87.9 & 68.2 & 48.6 & 56.8 \\
& Mismatch & 87.8 & 86.3 & 87.1 & 60.8 & 45.0 & 51.7 \\
\hline \multirow{2}{*}{ Four Datasets } & All Multi-task & 88.7 & 87.7 & 88.2 & 67.2 & 48.5 & 56.4 \\
& Multi-task DA & 89.7 & 88.3 & 89.0 & 60.2 & 52.3 & 59.9 \\
\hline
\end{tabular}

Table 3: Model variations grouped by number of training datesets.

section of Table 3, including additional training data - no matter from another task, domain or both - always improves the performance. A hidden factor not shown in the table is the additional dataset's size. For multi-task learning, since we are look at the social media domain, the additional dataset size is small. This is probably the reason why the Mismatch setting leveraging data from a different task and domain surprisingly outperformed multitask learning. Domain adaptation enjoys both the benefits of a large amount of additional training data and an aligned task, thus achieving the best results among the two dataset settings.

When conducting multi-task domain adaptation, we are leveraging four datasets. One concern is that the performance gains only come from additional training data, instead of the deliberately designed framework (Joshi et al., 2012). We thus also compare with a strategy which treats the same task for a different domain as a different task. The corresponding neural architecture is a shared BiLSTM with four separate task-specific models: we call it the All Multi-task setting. The results show that explicitly modeling data domains gives extra benefit than blindly throwing in more training data. We found the same benefits when experimenting with three datasets (instead of 2 or 4 ).

\section{Related Work}

The previous work on domain adaptation exclusively focused on building a unified model for $a$ task across domain. However, we argue that a flexible framework for domain adaptation on several tasks simultaneously would be beneficial. To the best of our knowledge, the work that is closest to ours is Yang and Hospedales (2015), which provided a unified perspective for multi-task learning and multi-domain learning (a more general case of domain adaptation) under the same perspective of representation learning. However, they only focused on exploring the common ground of multi- task learning and multi-domain learning, and did not explore the possibility of having multi-task learning to help domain adaptation. We briefly review previous work on domain adaptation and multi-task learning below.

\subsection{Domain Adaptation}

In domain adaptation, or more general multidomain learning, the goal is to learn a single model that can produce accurate predictions for multiple domains. An important characteristic of learning across domains is that each domain represents data drawn from a different distribution, yet share many commonalities. The larger the difference between these distributions, the larger the generalization error when learning across domains (BenDavid et al., 2010; Mansour et al., 2009).

As a result, a long line of work in multi-domain learning concerns learning shared representations, such as through identifying alignments between features (Blitzer et al., 2007, 2006), learning with deep networks (Glorot et al., 2011), using transfer component analysis (Pan et al., 2011), learning feature embeddings (Yang and Eisenstein, 2015) and kernel methods for learning low dimensional domain structures (Gong et al., 2012), among others. Another line sought for feature weight tying (Dredze and Crammer, 2008; Daumé III, 2007, 2009; Finkel and Manning, 2009; Kumar et al., 2010; Dredze et al., 2010) to transfer the learned feature weights across domains.

We combined the two lines and explored joint learning with multiple tasks.

\subsection{Multi-task Learning}

The goal of MTL (Caruana, 1997; Ando and Zhang, 2005) is to improve performance on different tasks by learning them jointly.

With recent progress in deep representation learning, new work considers MTL with neural networks in a general framework: learn a shared 
representations for all the tasks, and then a task specific predictor. The representations shared by tasks go from lower level word representations (Collobert and Weston, 2008; Collobert et al., 2011), to higher level contextual representations learned by Recurrent Neural Networks (RNNs) (Liu et al., 2016b; Yang et al., 2016; Peng et al., 2017) or other neural architectures (Liu et al., 2016a; Søgaard and Goldberg, 2016; Benton et al., 2017). MTL has helped in many NLP tasks, such as sequence tagging (Collobert et al., 2011; Peng and Dredze, 2016; Søgaard and Goldberg, 2016; Yang et al., 2016), text classification (Liu et al., 2016b,a), and discourse analysis (Liu et al., 2016c).

We expand the spectrum by exploring how multi-task learning can help domain adaptation.

\section{Conclusion}

We have presented a framework for multi-task domain adaptation, and instantiated a neural architecture for sequence tagging problems. The framework is composed of a shared representation learner for all datasets, a domain projection layer that learns one projection per domain, and a taskspecific model layer that learns one set of feature weights per task. The proposed neural architecture can be trained end-to-end, and achieved the stateof-the-art results for Chinese word segmentation and NER on social media domain.

With this framework in mind, there are several interesting future directions to explore. First, we considered common domain adaptation schemas with our domain mask and linear projection. However, there are many more sophisticated methods that we can consider integrating into our model (Blitzer et al., 2007; Yang and Eisenstein, 2015). Second, we only experimented with sequence tagging problems. However, the proposed framework is generally applicable to other problems such as text classification, parsing, and machine translation. We plan to explore these applications in the future. Finally, our work draws on two traditions in multi-domain learning: parameter sharing (on the task specific models) and representation learning (the shared representation learner). We plan to explore how other domain adaptation methods can be realized in a deep architecture.

\section{References}

Rie Kubota Ando and Tong Zhang. 2005. A framework for learning predictive structures from multiple tasks and unlabeled data. The Journal of Machine Learning Research .

Shai Ben-David, John Blitzer, Koby Crammer, Alex Kulesza, Fernando Pereira, and Jennifer Wortman Vaughan. 2010. A theory of learning from different domains. Machine learning .

Yoshua Bengio, Patrice Simard, and Paolo Frasconi. 1994. Learning long-term dependencies with gradient descent is difficult. IEEE Transactions on Neural Networks .

Adrian Benton, Margaret Mitchell, and Dirk Hovy. 2017. Multi-task learning for mental health using social media text. In Proceedings of EACL.

John Blitzer, Mark Dredze, Fernando Pereira, et al. 2007. Biographies, bollywood, boom-boxes and blenders: Domain adaptation for sentiment classification. In Proceedings of ACL.

John Blitzer, Ryan McDonald, and Fernando Pereira. 2006. Domain adaptation with structural correspondence learning. In Proceedings of EMNLP.

Rich Caruana. 1997. Multitask learning. Machine learning .

Rich Caruana, vSteve Lawrence, and Lee Giles. 2001. Overfitting in neural nets: Backpropagation, conjugate gradient, and early stopping. In Proceedings of NIPS.

Minmin Chen, Zhixiang Xu, Fei Sha, and Kilian Q Weinberger. 2012. Marginalized denoising autoencoders for domain adaptation. In Proceedings of $I C M L$.

Ronan Collobert and Jason Weston. 2008. A unified architecture for natural language processing: Deep neural networks with multitask learning. In Proceedings of ICML.

Ronan Collobert, Jason Weston, Léon Bottou, Michael Karlen, Koray Kavukcuoglu, and Pavel Kuksa. 2011. Natural language processing (almost) from scratch. The Journal of Machine Learning Research

James Cross and Liang Huang. 2016. Span-based constituency parsing with a structure-label system and provably optimal dynamic oracles. In Proceedings of EMNLP.

Hal Daumé III. 2007. Frustratingly easy domain adaptation. In Proceedings of $A C L$.

Hal Daumé III. 2009. Bayesian multitask learning with latent hierarchies. In Proceedings of UAI.

Mark Dredze and Koby Crammer. 2008. Online methods for multi-domain learning and adaptation. In Proceedings of EMNLP. 
Mark Dredze, Alex Kulesza, and Koby Crammer. 2010. Multi-domain learning by confidence-weighted parameter combination. Machine Learning .

Chris Dyer, Miguel Ballesteros, Wang Ling, Austin Matthews, and Noah A Smith. 2015. Transitionbased dependency parsing with stack long shortterm memory. In Proceedings of ACL.

Thomas Emerson. 2005. The second international chinese word segmentation bakeoff. In Proceedings of the fourth SIGHAN workshop on Chinese language Processing.

Jenny Rose Finkel and Christopher D Manning. 2009. Hierarchical bayesian domain adaptation. In Proceedings of NAACL.

Xavier Glorot, Antoine Bordes, and Yoshua Bengio. 2011. Domain adaptation for large-scale sentiment classification: A deep learning approach. In Proceedings of ICML.

Boqing Gong, Yuan Shi, Fei Sha, and Kristen Grauman. 2012. Geodesic flow kernel for unsupervised domain adaptation. In Proceedings of CVPR.

Alan Graves, Abdel-rahman Mohamed, and Geoffrey Hinton. 2013. Speech recognition with deep recurrent neural networks. In Proceedings of ICASSP.

Sepp Hochreiter and Jürgen Schmidhuber. 1997. Long short-term memory. Neural computation .

Mahesh Joshi, William W Cohen, Mark Dredze, and Carolyn P Rosé. 2012. Multi-domain learning: when do domains matter? In Proceedings of EMNLP.

Eliyahu Kiperwasser and Yoav Goldberg. 2016. Simple and accurate dependency parsing using bidirectional LSTM feature representations. Transactions of the Association for Computational Linguistics .

Abhishek Kumar, Avishek Saha, and Hal Daume. 2010. Co-regularization based semi-supervised domain adaptation. In Proceedings of NIPS.

John Lafferty, Andrew McCallum, and Fernando CN Pereira. 2001. Conditional random fields: Probabilistic models for segmenting and labeling sequence data. In Proceedings of ICML.

Guillaume Lample, Miguel Ballesteros, Sandeep Subramanian, Kazuya Kawakami, and Chris Dyer. 2016. Neural architectures for named entity recognition. In Proceedings of NAACL.

Gina-Anne Levow. 2006. The third international chinese language processing bakeoff: Word segmentation and named entity recognition. In Proceedings of the Fifth SIGHAN Workshop on Chinese Language Processing.

Pengfei Liu, Xipeng Qiu, and Xuanjing Huang. 2016a. Deep multi-task learning with shared memory. In Proceedings of EMNLP.
Pengfei Liu, Xipeng Qiu, and Xuanjing Huang. 2016b. Recurrent neural network for text classification with multi-task learning. In Proceedings of IJCAI.

Yang Liu, Sujian Li, Xiaodong Zhang, and Zhifang Sui. 2016c. Implicit discourse relation classification via multi-task neural networks. In Proceedings of AAAI.

Xuezhe Ma and Eduard Hovy. 2016. End-to-end sequence labeling via bi-directional lstm-cnns-crf. In Proceedings of ACL.

Yishay Mansour, Mehryar Mohri, and Afshin Rostamizadeh. 2009. Domain adaptation: Learning bounds and algorithms. In Proceedings of COLT.

Quinn McNemar. 1947. Note on the sampling error of the difference between correlated proportions or percentages. Psychometrika 12(2):153-157.

Sinno Jialin Pan, Ivor W Tsang, James T Kwok, and Qiang Yang. 2011. Domain adaptation via transfer component analysis. IEEE Transactions on Neural Networks .

Razvan Pascanu, Tomas Mikolov, and Yoshua Bengio. 2013. On the difficulty of training recurrent neural networks. In Proceedings of ICML.

Nanyun Peng and Mark Dredze. 2015. Named entity recognition for chinese social media with jointly trained embeddings. In Proceedings of EMNLP. Lisboa, Portugal.

Nanyun Peng and Mark Dredze. 2016. Improving named entity recognition for chinese social media with word segmentation representation learning. In Proceedings of ACL.

Nanyun Peng, Hoifung Poon, Chris Quirk, Kristina Toutanova, and Wen-tau Yih. 2017. Cross-sentence n-ary relation extraction with graph lstms. Transactions of the Association for Computational Linguistics 5:101-115.

Anders Søgaard and Yoav Goldberg. 2016. Deep multi-task learning with low level tasks supervised at lower layers. In Proceedings of the 54th Annual Meeting of the Association for Computational Linguistics. Association for Computational Linguistics, volume 2, pages 231-235.

Yan Xu, Lili Mou, Ge Li, Yunchuan Chen, Hao Peng, and Zhi Jin. 2015. Classifying relations via long short term memory networks along shortest dependency paths. In Proceedings of EMNLP.

Yi Yang and Jacob Eisenstein. 2015. Unsupervised multi-domain adaptation with feature embeddings. In Proceedings of NAACL.

Yongxin Yang and Timothy M Hospedales. 2015. A unified perspective on multi-domain and multi-task learning. Proceedings of ICLR . 
Zhilin Yang, Ruslan Salakhutdinov, and William Cohen. 2016. Multi-task cross-lingual sequence tagging from scratch. arXiv preprint arXiv:1603.06270

Longkai Zhang, Li Li, Zhengyan He, Houfeng Wang, and Ni Sun. 2013. Improving chinese word segmentation on micro-blog using rich punctuations. In Proceedings of ACL.

Shu Zhang, Dequan Zheng, Xinchen Hu, and Ming Yang. 2015. Bidirectional long short-term memory networks for relation classification. In Proceedings of 29th Pacific Asia Conference on Language, Information and Computation. 